\title{
Acute Lymphoblastic Leukemia masquerading as surgical third nerve palsy: a case report
}

\author{
Ting Jacqueline YL*, Ho Niki WW and Pan Shin-Wei \\ Ophthalmology Department, Sibu Hospital, Malaysia
}

\begin{abstract}
Purpose: To describe a case of Acute Lymphoblastic Leukemia masquerading as surgical third nerve palsy.

Method: This is a case report of a 50-years old gentleman presented with a one week history of sudden right eye ptosis with headache and vomiting. Complete ptosis was noted over the right eye with a positive relative afferent pupillary defect (RAPD) over the affected eye. There was a restricted medial, superior and inferior gaze movement. Funduscopy was normal with no evidence of papilloedema. Urgent Computed tomography (CT) Brain was done to rule out surgical cause of third nerve palsy; but came back normal. Patient subsequently came back one month later with left eye ptosis in addition to the unresolved right eye ptosis. Cranial nerve examination depicted bilateral $3^{\text {rd }}$ cranial nerve palsy and right $7^{\text {th }}$ cranial nerve palsy.
\end{abstract}

Results: Routine blood investigations and blood smear showed presence of hyperleukocytosis with 90\% abnormal cells. Bone marrow aspirate and trephine (BMAT) was done and result depicted T-cell Acute Lymhoblastic Leukemia (T-ALL). He was referred to a haematologist and started on chemotherapy.

Conclusion: Acute lymphoblastic leukemia is not very common and diagnosis is a challenge due to its uncommon presentation. Surgical oculomotor nerve palsy usually necessitates neuro-imaging to rule out life-threatening aneurysms and tumours. However, other uncommon causes such as central nervous system infiltration by leukemia may also occur. Proper history and physical examination, and laboratory blood investigations would aid in coming to an accurate diagnosis.

\section{Introduction}

Acute lymphoblastic leukemia (ALL) is a haematological malignancy that can involve the central nervous system (CNS). Less than $10 \%$ of patients with ALL have CNS involvement at presentation. We report a very unusual case of ALL, masquerading as surgical third nerve palsy at presentation, and discuss the assessment, diagnosis and management of this patient.

\section{Case report}

A 50-years old gentleman presented with a one-week history of sudden right eye ptosis with headache and vomiting. He did not have any fever and other systemic symptoms. His past ocular and medical history was insignificant. On ocular examination his best corrected visual acuity was 6/12 in the right eye and 6/9 in the left eye. Intraocular pressure (IOP) on applanation tonometry was $12 \mathrm{mmHg}$ bilaterally. Complete ptosis was noted over the right eye with a positive relative afferent pupillary defect (RAPD) over the affected eye. The pupil was also mid dilated; $5 \mathrm{~mm}$ but was reactive to light. There was a restricted medial, superior and inferior gaze movement, which was highly suggestive of third cranial nerve palsy. There was no proptosis. Funduscopy was normal with no evidence of papilloedema. Ocular examination over the fellow eye was unremarkable. Based on his systemic complaints and clinical findings, an urgent Computed Tomography (CT) Brain was done to rule out surgical cause of third nerve palsy. Routine blood investigations including white cell count $11.4 \times 10^{6} / \mu \mathrm{L}$ and CT Brain was normal with no evidence of intracranial bleeding or space-occupying lesion.

Patient subsequently came back one month later with sudden onset of left eye ptosis in addition to the unresolved right eye ptosis. The left pupil was dilated at $6 \mathrm{~mm}$ while the fellow eye's pupil was of a normal size. RAPD was found to be negative. During this presentation, he also had lower motor neuron facial nerve palsy over the right side with loss of forehead and brow movements, inability to close eyes and drooping of eyelids, loss of nasolabial folds and drooping of lower lip. Cranial nerve examination depicted bilateral $3^{\text {rd }}$ cranial nerve palsy and right $7^{\text {th }}$ cranial nerve palsy. Trochlear nerve, Trigeminal nerve and abducens nerve function was intact. Due to multiple cranial nerve involvement, a repeated CT brain and orbit was done. However, the result of which was also normal. Blood investigations revealed a white blood count $(\mathrm{WBC})$ of $105 \times 10^{6} / \mu \mathrm{L}$ (Normal range $\left.=4-10 \times 10^{3} / \mu \mathrm{L}\right)$ and peripheral blood smear showed presence of hyperleukocytosis with $90 \%$ abnormal cells which are heterogenous in sizes, have scanty cytoplasm, slightly clumped homogenous nuclear chromatin and inconspicuous nucleoli. Patient was advised for bone marrow aspirate and trephine (BMAT) biopsy and sent for immunophenotyping. Result depicted T-cell Acute Lymhoblastic Leukemia (T-ALL). He was referred to a haematologist and started on chemotherapy.

\section{Discussion}

Acute lymphoblastic leukemia is not very common, with a crude incidence rate of 1 new ALL case for every 100,000 male patients, and

*Correspondence to: Ting Jacqueline YL, Ophthalmology Department, Sibu Hospital, Batu 5 1/2 Ulu Oya Road 96000 Sibu, Sarawak, Malaysia, Tel: +6084343333 Ext 1004/1002; Fax: +6084337354; E-mail: jac6198@hotmail.com

Key words: acute lymphoblastic leukemia, third nerve palsy, haematological malignancy, central nervous system involvement

Received: June 12, 2018; Accepted: June 25, 2018; Published: June 29, 2018 
less than 1 for every 100,000 female patients in UK [1]. The T-cell variant accounts for $25 \%$ of acute lymphoblastic leukemias in adults [2]. Its clinical presentation is a challenge due to its uncommon and aggressive presentation. Presentation can include symptoms associated with hyperleukocytosis, with lymphadenopathy and extramedullary organ involvement. Central nervous system infiltration is also quite common. [3]

Acute paresis of the oculomotor nerve with pupillary involvement warrants neuro-imaging to rule out aneurysms and tumours. This clinical manifestation may be due to a ruptured aneurysm, which may herald subarachnoid hemorrhage and death. In $92 \%$ of patients with tumour, and in $78.5 \%$ of patients with aneurysm, the pupil was fixed and dilated on examination [4]. For this particular patient, we did a CT scan twice, due to a presentation with surgical $3^{\text {rd }}$ nerve palsy. However, both the initial and repeated imaging was negative.

Most causes of multiple cranial nerve palsies are detectable via neuro-imaging. However, leukemic infiltration of the central nervous system may not be detectable on computed tomography. In a study by JJ Pagani et al, CT was normal in 374 patients including 148 with meningeal disease diagnosed by cerebrospinal fluid cytologic examination. Only 31 out of 405 had computed tomographic abnormalities in the brain. CT detection of leukemia using both conventional and delayed high dose scans, the meninges and cisternal spaces were normal in all patients in the present series with known diffuse meningeal disease. The intense meningeal staining seen with carcinomatous or infectious meningitis occurs only rarely [5]. Furthermore, a more superior imaging modality would be magnetic resonance imaging as it gives high soft-tissue contrast and ability to show the entire course of the third nerve. CT scanning is limited in patients with spontaneous subarachnoid haemorrhage, or tumours [6].

We report this case of ALL presenting as surgical third nerve palsy as we acknowledge that the incidence is rare and the due to its atypical presentation, diagnosis is a challenge. In addition, we would like to emphasize the importance of thorough history and physical examination, appropriate laboratory blood investigations and imaging modalities. In surgical third nerve palsies, common lifethreatening etiologies such as a ruptured cerebral aneurysm should be ruled out, but other uncommon causes such as central nervous system infiltration by leukemia should also be kept in mind. (Figures 1 and 2)

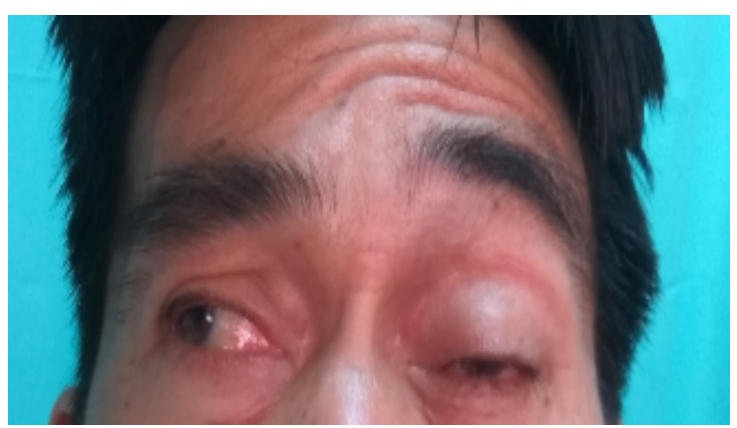

Figure 1. Right eye mild ptosis, left eye severe ptosis with loss of forehead and brow movements.

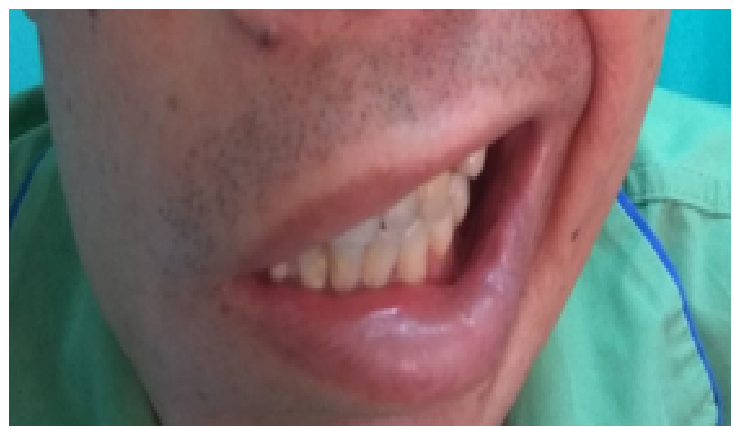

Figure 2. Right sided loss of nasolabial folds and drooping of lower lip

\section{Acknowledgement}

We thank the patient for allowing us to report his case.

\section{References}

1. Cancer Research UK (2017) http://www.cancerresearchuk.org/health-professional/ cancer-statistics/statistics-by-cancer-type/leukaemia-all/incidence\#heading-Zero, Assessed [July]

2. Chiaretti S, Foà R (2009) T-cell acute lymphoblastic leukemia. Haematologica 94: 160 162. [Crossref]

3. Litzow MR, Ferrando AA (2015) How I treat T-cell acute lymphoblastic leukemia in adults. Blood 126: 833-841. [Crossref]

4. Singh A, Bahuguna C, Nagpal R, Kumar B (2016) Surgical management of third nerve palsy. Oman J Ophthalmol 9: 80-86. [Crossref]

5. Pagani, JJ, Libshitz, HI, Wallace, S, and Hayman, LA (1981) Central nervous system leukemia and lymphoma: computed tomographic manifestations. AJR 137: 1195-1201

6. Lo CP, Huang CF, Hsu CC, Kuo CC, Liu CC, et al. (2012) Neuroimaging of isolated and non-isolated third nerve palsies. Br J Radiol 85: 460-467. [Crossref]

Copyright: (C2018 Ting Jacqueline YL. This is an open-access article distributed under the terms of the Creative Commons Attribution License, which permits unrestricted use, distribution, and reproduction in any medium, provided the original author and source are credited. 